\title{
MiRNA-130a promotes inflammation to accelerate atherosclerosis via the regulation of proliferator-activated receptor y (PPAR $)$ expression
}

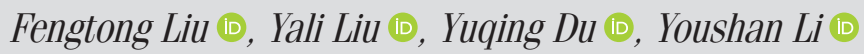

Department of Peripheral Vascular, Dongzhimen Hospital, Beijing University of Chinese Medicine; Beijing-China

\section{ABSTRACT}

Objective: In this study, we aimed to evaluate the possible function of miR-130a in atherosclerosis (AS), protection against AS, and its molecular biological mechanism.

Methods: Apoe-/- mice were fed a high-fat diet as the AS mice model. Human umbilical vein endothelial cells (HUVECs) were used as in vitro model. Serum samples or cells were used to measure the expression of inflammation. Serum samples or cells were used to determine MiRNA expression profiles using the edgeR tool from Bioconductor. Western Blot analysis was used to assess protein expressions of proliferatoractivated receptor у (PPARү) and nuclear factor (NF)-кВ.

Results: MiRNA-130a expression was up-regulated in atherosclerotic mice. In addition, over-expression of miRNA-130a promoted inflammation factors [tumor necrosis factor (TNF)- ${ }_{\alpha}$ and interleukin (IL)- ${ }_{\beta}$, IL-6, and IL-8] in the in vitro model of AS. However, down-regulation of miRNA130a reduced inflammation (suppressed TNF- $\alpha$, IL-1 $\beta$, IL-6 and IL-8) in the in vitro model. Furthermore, over-expression of miRNA-130a could also suppress the protein expression of PPARy and induce NF-кB protein expression in the in vitro model. However, suppression of miRNA-130a induced the protein expression of PPARy and suppressed NF-кB protein expression in the in vitro model of AS. Activation of PPARy reduced the pro-inflammatory effects of miRNA-130a on the AS-induced in vitro model.

Conclusion: These results strongly support that miRNA-130a suppression can protect against atherosclerosis through inhibiting inflammation by regulating the PPARy/ NF-кB expression.

Keywords: microRNA-130a, atherosclerosis, inflammation, PPAR-gamma, NF-kappaB

Cite this article as: Li Y, Liu F, Liu Y, Du Y. MiRNA-130a promotes inflammation to accelerate atherosclerosis via the regulation of proliferator-activated receptor Y (PPARY) expression. Anatol J Cardiol 2021; 25: 630-7.

\section{Introduction}

Atherosclerosis (AS) can be induced by multiple factors such as hyperlipidemia, hypertension, and smoking (1). Currently, the inflammation theory based on lipid infiltration theory and injury response theory is extensively recognized to be the pathogenesis of AS (2). First proposed by the famous scholar Ross in 1976, this theory believes that AS is a process of pathological change in which lipid accumulates in the arterial wall to form a local plaque. It is mediated by inflammatory response accompanied by the genesis of oxidative stress (OS) (3). The interactions between the immune inflammatory cells (such as macrophages and dendritic cells) and multiple inflammatory factors play a key role in AS genesis and development, which can be ascribed to the characteristics of multiple targets and complexity of inflammatory action of cytokines (2).
AS is a chronic inflammatory disease induced by multiple factors in the vascular wall (4). Studies on the regulatory effect of miRNA during AS development are ongoing (4). Nonetheless, it is believed that miRNA expression in the vascular wall cells (endothelial and smooth muscle cells) as well as the infiltrated white blood cell subsets can affect disease development (5). Typically, the role of miRNA in regulating cell growth, differentiation, proliferation, and apoptosis is being gradually discovered. Moreover, abnormal miRNA expression is verified to be closely correlated with the genesis and development of multiple diseases (6). In recent years, the role of miRNA in AS has attracted increasing attention (6). Li et al. (7) have indicated that miR-130a has a role in the regulation of tumor necrosis factor (TNF) a in osteoarthritis. Yao et al. (8) have shown that the knockdown of miR-130a-3p alleviates NF-кB in spinal cord injury.

Address for Correspondence: Dr. Youshan Li, Department of Peripheral Vascular, Dongzhimen Hospital, Beijing University of Chinese Medicine; Beijing-China Phone: +86-010-84013346 E-mail: liyoushan11@yeah.net

Accepted Date: 24.03.2021 Available Online Date: 16.08.2021

(C) Copyright 2021 by Turkish Society of Cardiology - Available online at www.anatoljcardiol.com DOI:10.5152/AnatolJCardiol.2021.56721 


\section{HIGHLIGHTS}

- MiRNA-130a expression was up-regulated in atherosclerotic mice;

- MiRNA-130a promotes inflammation in model of atherosclerosis;

- MiRNA-130a accelerates atherosclerosis;

- MiRNA-130a inhibits inflammation by the induction of PPARy to suppress NF- $\mathrm{kB}$ expression in model of atherosclerosis

Peroxisome proliferator-activated receptor (PPAR), which is a ligand-activated transcription factor, is a member of the nuclear receptor superfamily that can react with specific DNA response elements to regulate gene expression (9). Its main function is converting the homeostasis changes, drugs, nutrition, and inflammatory stimulations in the body into intracellular signals (10). Thus, it plays a key role as a messenger in regulating energy metabolism, cell differentiation, proliferation, apoptosis, inflammatory response, endogenous active substance synthesis, and secretion (9). Meanwhile, PPAR can be activated by endogenous fatty acids and its metabolites and is, therefore, named the fatty acid receptor (11). PPARy is a subtype of PPAR whose vascular and biological functions gradually starts with the discovery of PPARy expression in mononuclear macrophages, endothelial cells, and vascular smooth muscle cells (9).

Nuclear factor-кB (NF-кB) is a transcription factor that can regulate multiple gene transcription functions and specifically bind with multiple specific sites in cell gene promoter or enhancer sequences (12). Therefore, it can promote gene transcription and expression and is closely related to important pathophysiological processes (13). Recent studies indicate that genes involved in the inflammatory response during atherosclerotic plaque formation are mostly the NF-кB target genes, whose transcription and expression are mainly regulated by the NF-кB/IкB signaling pathway (13). Wang et al. (14) have reported that miR-130a upregulates the mTOR pathway by NF-кB in high-grade serous ovarian carcinoma. In this study, we aimed to evaluate the mechanism and function of miR-130a in atherosclerosis and inflammation and appraised its possible molecular biological I.

\section{Methods}

\section{Atherosclerosis animal model}

The animal experiment was approved by the Animal Health and Utilization Committee of the Beijing University of Traditional Chinese Medicine. ApoE-/- mice were fed with a high-fat diet for 12 weeks similar to the AS model group in literature (15). C57BL/6 mice (WT, $n=6$ ) were fed a normal diet for 12 weeks.

\section{Enzyme-linked immunosorbent assay}

After induction model of AS, all the mice were sacrificed by decapitation after anesthetizing them with $35 \mathrm{mg} / \mathrm{kg}$ pentobarbital sodium. Whole blood of all the mice was used to collect serum samples at $1000 \mathrm{~g}$ for $10 \mathrm{~min}$ at $4^{\circ} \mathrm{C}$, and the serum samples were saved at $-80^{\circ} \mathrm{C}$ to measure the expression of inflammation levels using enzyme-linked immunosorbent assay (ELISA) kits. T TNF- $\alpha$, IL-1 $\beta$, IL-6 and IL-18 were measured using TNF-a (H052), IL-1 $\beta$ (H002), IL-6 (H007) and IL-18 (H015) ELISA kits (Nanjing Jiancheng Biology Engineering Institute).

\section{Oil Red 0 staining}

The blood vessels were collected and fixed with $4 \%$ paraformaldehyde for one day. The aorta was embedded in paraffin and cut into $5 \mu \mathrm{M}$ sections on the vibrator. The aorta was then stained with hematoxylin and eosin for 15 minutes and measured with a Olympus microscope (Olympus optics, Tokyo, Japan).

\section{Reverse transcription quantitative PCR (RT-qPCR) and miR- NA expression profiles}

Total RNA was extracted from cells with TRIzol reagent. The cDNA was reverse transcripted by Primescript Kit (Takara, Dalian, China), and real-time quantitative PCR was performed using 7500 real-time PCR system (Applied Biological Systems, Forster, California, USA) Power SYBR Green Master Mix (Takara, Dalian, China). RT-PCR was carried out as follows: Pre denaturation at $94^{\circ} \mathrm{C}$ for 10 minutes, denaturation at $94^{\circ} \mathrm{C}$ for 30 seconds, annealing at $60^{\circ} \mathrm{C}$ for 30 seconds, extension at $72^{\circ} \mathrm{C}$ for two minutes, 40 cycles. ORT PCR primers: miRNA-130a, 5 '- GTCAGTGCTAAAAGGGCAT-3' and reverse, 5 '- CAGTGCGTGTCGTGGAGT-3'; U6 forward, 5 '- GCTTCGGCAGCACTATAAT-3' and reverse, 5 'CGCTTCACGAATTGCTGTCAT-3’. The relative gene expression was calculated by $2-\Delta \Delta \mathrm{Ct}$ method (16).

RNA samples were amplified by ovation PicoSL WTA System V2 Kit (NuGEN) and sureprint G3 human Ge V2, 8x60k microarray (Agilent). cDNAs was produced using superscript II reverse transcriptase (Biotechnology). Surescan microarray scanner was used for data acquisition, and feature extraction software $v$. 10.7.3.1 (Agilent, USA) was used to extract the signal, which was submitted to GEO (gene expression integrated system, registration number gse80754).

\section{Human umbilical vein endothelial cells in vitro model of atherosclerosis}

Human umbilical vein endothelial cells (HUVECs) were cultured in Dulbecco's modified Eagle's medium (DMEM) supplemented with $10 \%$ fetal bovine serum (FBS) (Thermo Fisher Scientific, MA, USA) at $37^{\circ} \mathrm{C}$ in an atmosphere containing $5 \%$ carbon dioxide $\left(\mathrm{CO}_{2}\right)$. MiRNA-130a (supplemented with 10\% FBS), antimiRNA-30a (anti-miRNA-130a with 10\% FBS), and negative mimics (negative mimics with $10 \%$ FBS) were transfected into HUVECs using Lipofectamine 2000 (Thermo Fisher Scientific, Inc.). HUVECs were transfected with negative mimics in the negative group, with miRNA-130a mimics in the miRAN-130a group, and with anti-miRNA-130a mimics in the anti-130a group. After trans- 
fection at $37^{\circ} \mathrm{C}$ for six hours, the medium of all the groups was subsequently replaced with DMEM containing 10\% FBS for 42 hours and treated with $100 \mathrm{ng}$ lipopolysaccharide (LPS) 2000 for six hours. The cell supernatant medium was collected at 2000 $\mathrm{g}$ for 10 minutes at $4^{\circ} \mathrm{C}$, and the level of inflammation was measured by ELISA.

\section{Dual-luciferase reporter assay}

According to the bioinformatics results, the 3 '-untranslated region (UTR) of PPARy was cloned into pMIR-REPORT luciferase reporter plasmids (Promega Corporation, Madison, WI, USA). PPARy plasmids were co-transfected with miR-24 mimics into HUVECs using Lipofectamine 2000 . After incubation at $37^{\circ} \mathrm{C}$ for 48 hours, the cells were lysed using a dual luciferase reporter assay kit (Promega Corporation).

\section{Western blot analysis}

According to the manufacturer's protocol, total protein was extracted by Ripa analysis (p0013b, Beyotime) and quantified by BCA Kit (p0009, Beyotime). A total of $50 \mathrm{~g}$ of protein was placed on $12 \%$ sodium alkylsulfate polyacrylamide gel and transferred to a polyvinylidene difluoride membrane. The membrane was sealed in 5\% skim milk for one hour at room temperature and incubated with the main antibody overnight at $4^{\circ} \mathrm{C}$ : PPARy (sc166731, 1:1000, Santa Cruz biotechnology group), NF-кB (sc71677, 1:1000, Santa Cruz biotechnology group), and GAPDH (sc51631, 1:5000, Santa Cruz biotechnology group). Subsequently, the membrane was washed with tris-buffered saline and Polysorbate 20 (TBST) and incubated with horseradish peroxidase bound goat anti-rabbit IgG at room temperature for one hour (sc2004, 1:5000, Santa Cruz Biotechnology). Protein detection was performed by enhanced chemiluminescence (ECL, Beijing Beyotime Institute of Biotechnology, China), and imaging was performed by sodium imaging laboratory 3.0 (Bio-Rad Laboratories).

\section{Immunofluorescence}

The cells were washed with phosphate-buffered saline (PBS) for 15 minutes and fixed with 4\% paraformaldehyde for 15 minutes. The cells were connected with PPARy (Sc-

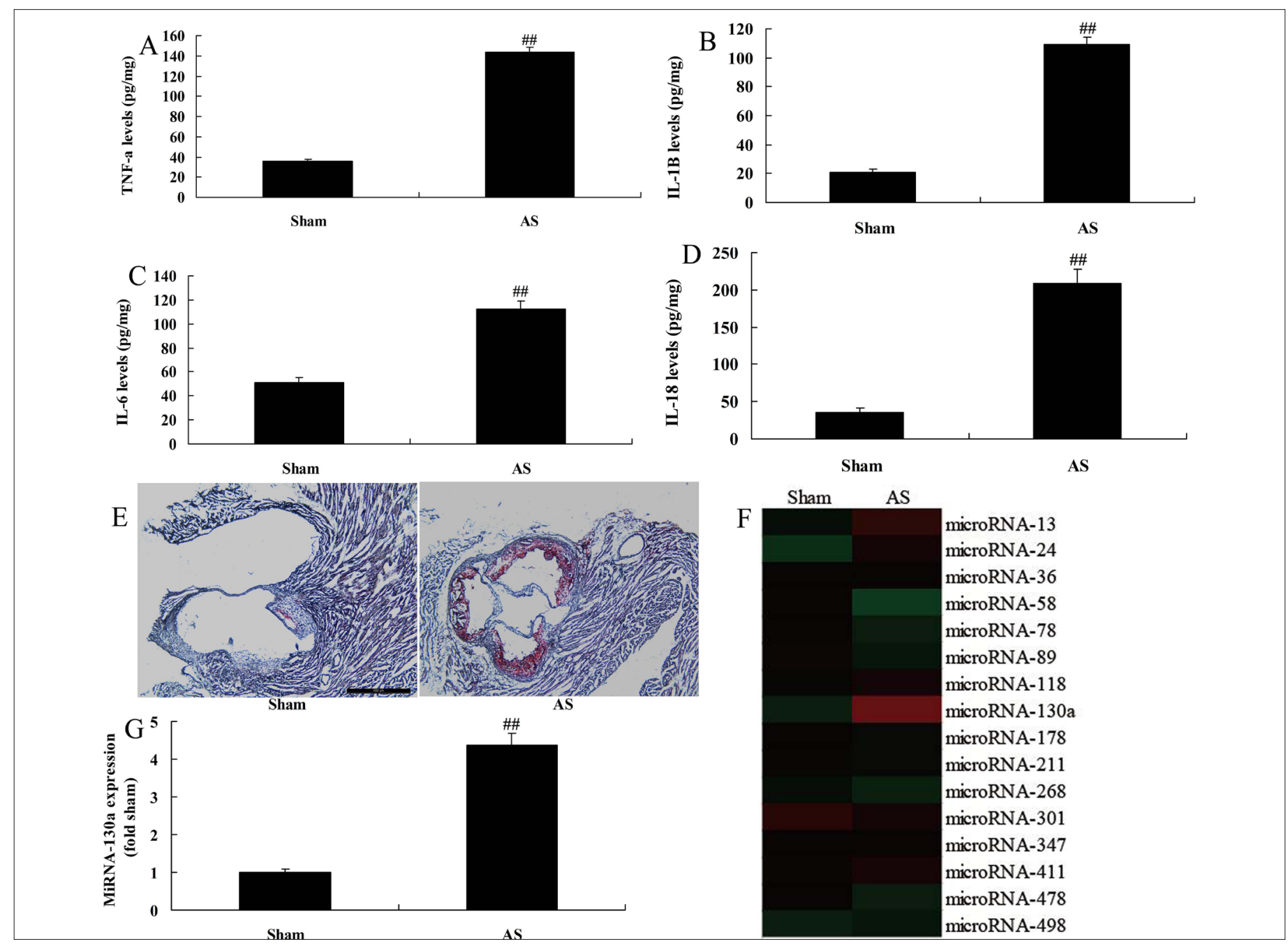

Figure 1. Expression of miRNA-130a in atherosclerotic mice A, B, C and D: TNF- $\alpha$, IL-1 $\beta$, IL-6, and IL-18 levels

$F$ and G: Oil Red 0 staining $(\times 100, E)$, gene chip and qPCR for miRNA-130a expression. Sham group; AS, miRNA-130a group. $\# P<0.01$ comparison with sham control group. 


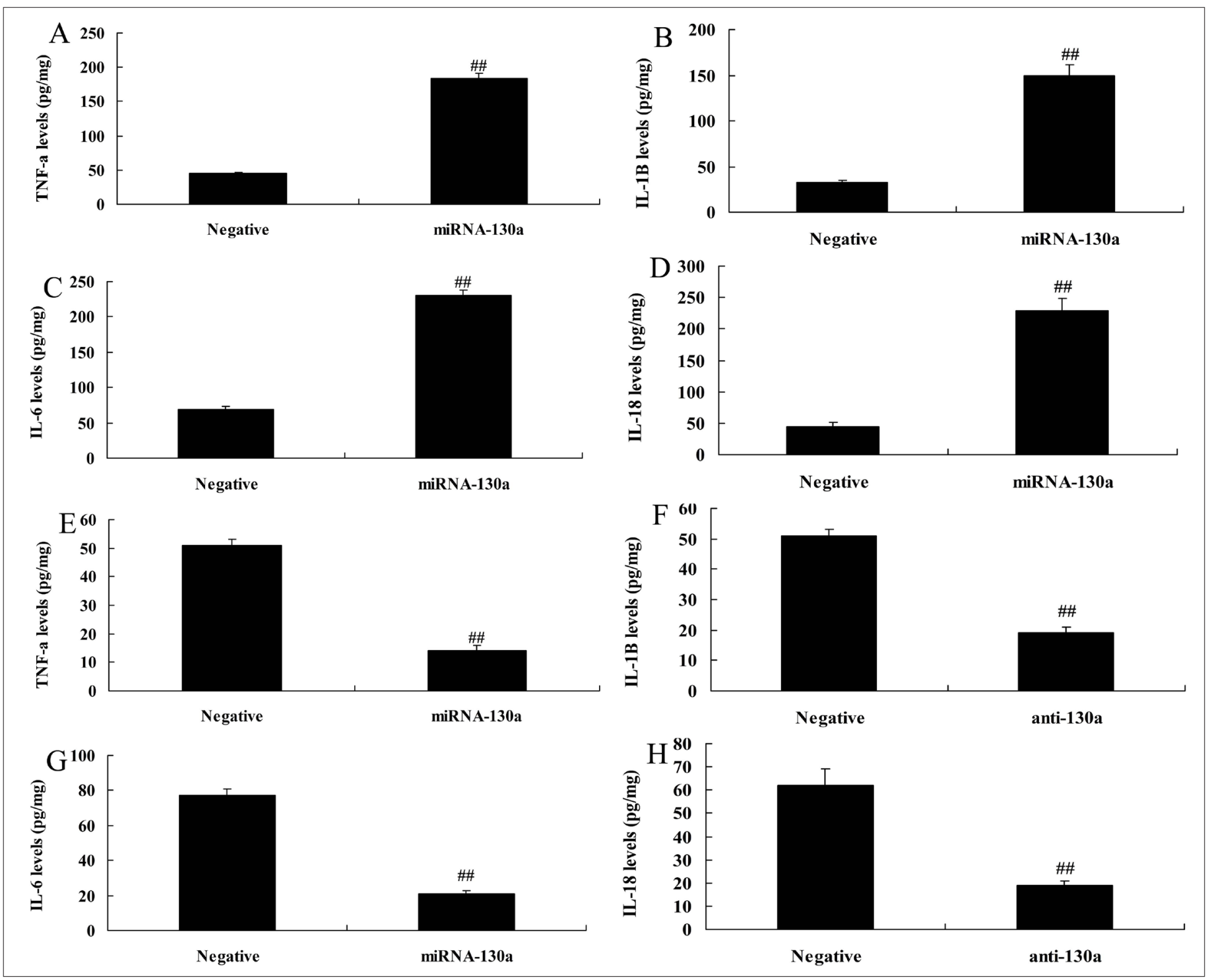

Figure 2. MiRNA-130a regulates inflammation in in vitro human umbilical vein endothelial cells A, B, C and D: TNF-a, IL-1 1 , IL-6, IL-18 levels by over-expression of miRNA-130a

$\mathrm{E}, \mathrm{F}, \mathrm{G}$ and $\mathrm{H}: \mathrm{TNF}-\mathrm{a}, \mathrm{IL}-1 \beta, \mathrm{IL}-6, \mathrm{IL}-18$ levels by down-regulation of miRNA-130a. Negative, negative control group; miRNA-130a, over-expression of miRNA-130a group; anti-130a, down-regulation of miRNA-130a group. $\# P<0.01$ comparison with negative group.

166731, 1:100, Santa Cruz biotechnology company) and $0.25 \%$ Trionx-100 were incubated overnight in PBS at $4^{\circ} \mathrm{C}$. The cells were incubated with goat anti-rabbit IgG CFL 555 (sc-362272, $1: 100$, Santa Cruz biotechnology company) at $37^{\circ} \mathrm{C}$ for two hours and stained with 4',6-diamidino-2-phenylindole in the dark for 15 minutes. Cells were observed using Zeiss Axioplan 2 (Carl Zeiss Microlmaging).

\section{Statistical analysis}

All data were expressed as mean \pm standard deviation $(n=3)$ using the Statistical Package for the Social Sciences version 18.0 software (SPSS Inc., Chicago, Illinois, USA). The data between the groups were compared by student's t test or one-way analysis of variance and post-ho test. $P<0.05$ was considered to be statistically significant.

\section{Results}

miRNA-130a serum levels expression in atherosclerotic mice

The serum expression levels of IL-18, IL-1 $\beta$, TNF-a, and IL-6 were increased in the atherosclerotic mice model compared with those in the sham group (Fig. 1a-1d). Oil Red 0 staining suggested that the rate of thrombus in the atherosclerotic mice was higher than that in the sham group (Fig. 1e). In addition, the heat map or qPCR demonstrated that miRNA-130a expression was upregulated in atherosclerotic mice than that in the sham group (Fig. 1e-1g).

MiRNA-130a regulated inflammation in LPS-induced HUVECs in vitro model of AS

MiRNA-130a over-expression was found to increase the levels of TNF- $a$, IL-1 $\beta$, IL-6, and IL-18 in HUVECs in LPS-induced 


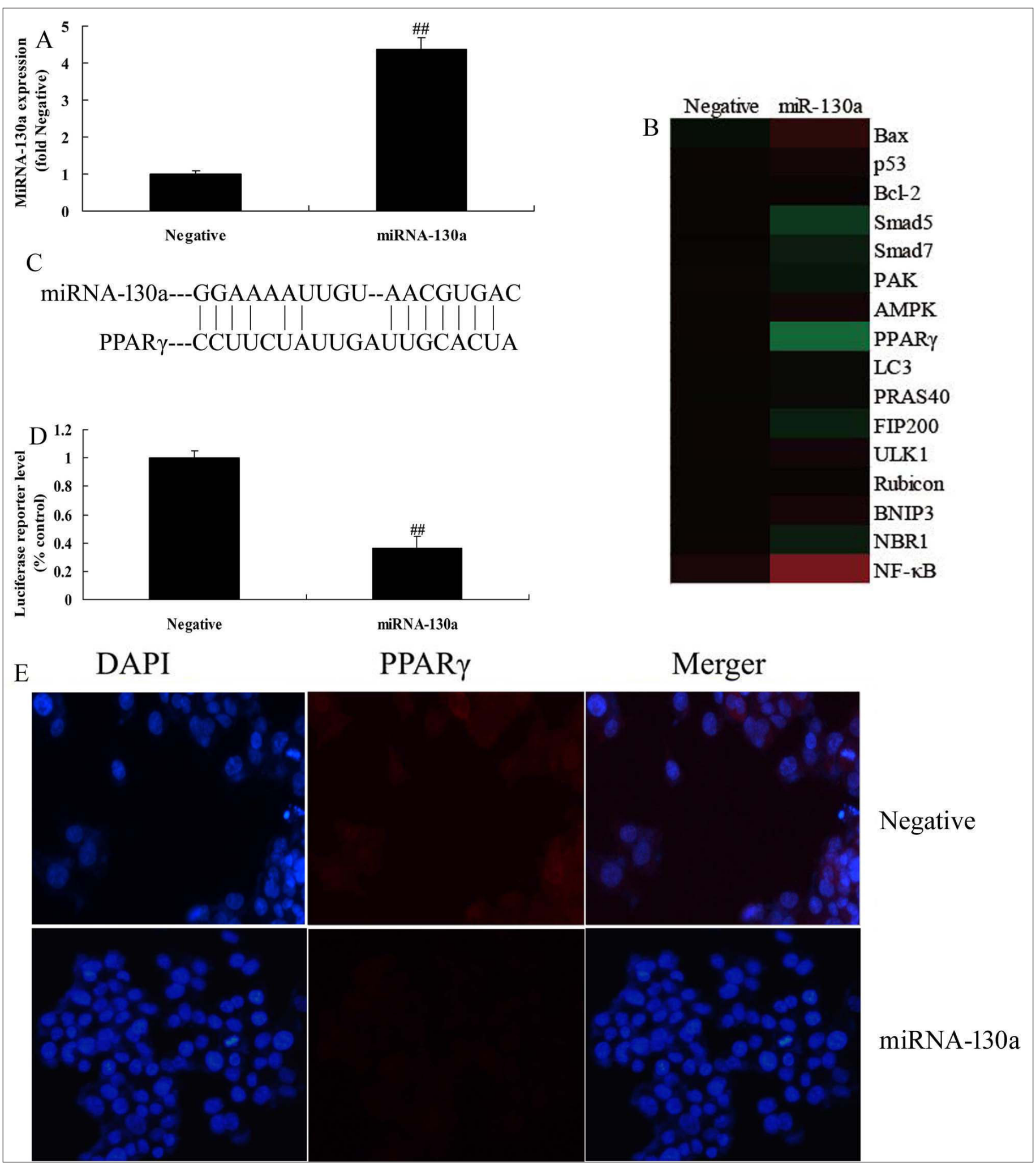

Figure 3. Over-expression of miRNA-130a regulates $P P A R y / N F-\kappa B$ in in vitro human umbilical vein endothelial cells A: $q P C R$ for miRNA-130a expression

B: gene chip

C, D, and E: $3^{\prime}$-UTR of PI3K is a direct target site for miRNA-130a, luciferase activity levels, and PPARy expression Negative, negative control group; miRNA-130a, over-expression of miRNA-130a group. $\# P<0.01$ comparison with negative group. 


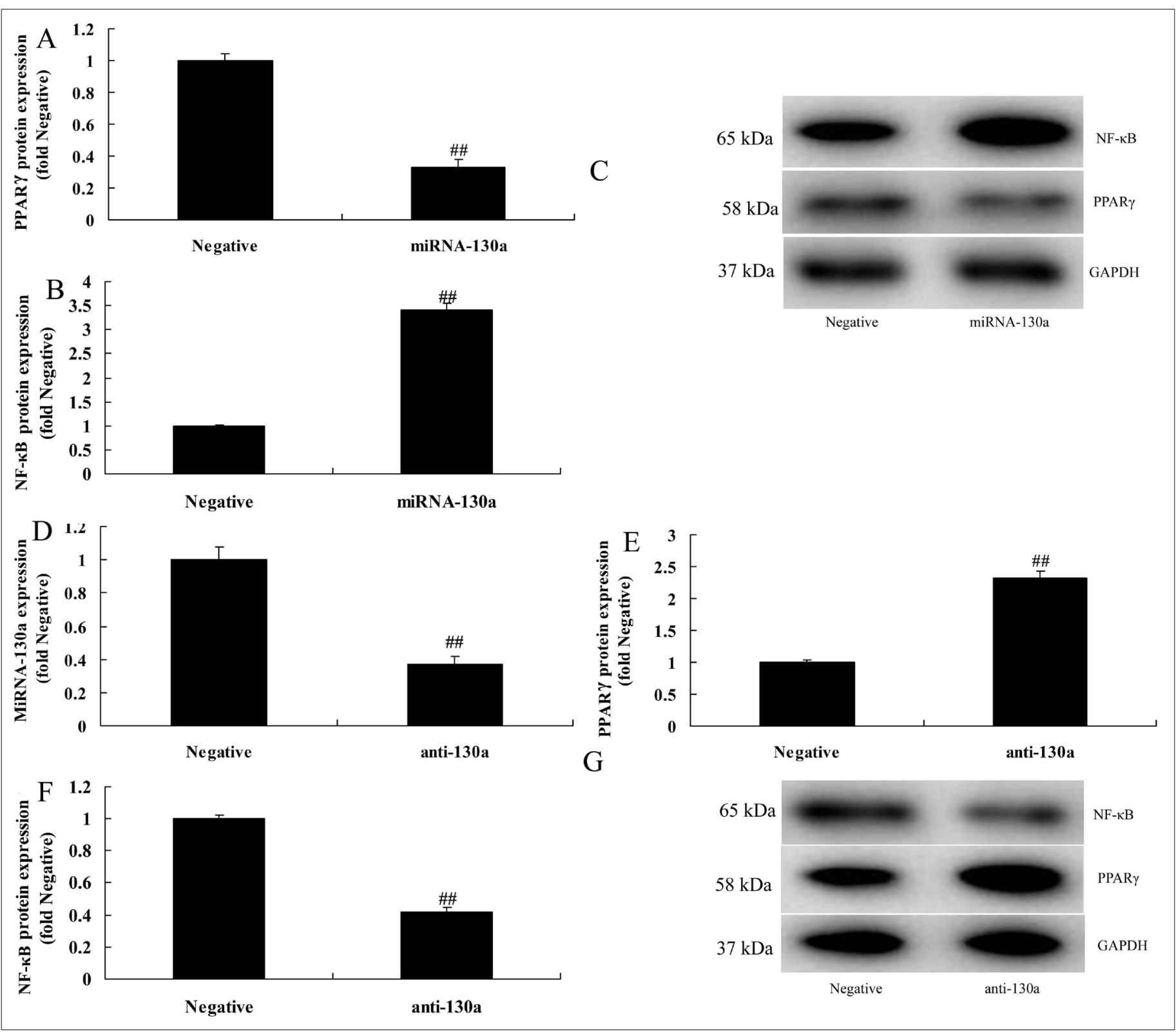

Figure 4. MiRNA-130a regulates PPARy/NF-кB in in vitro human umbilical vein endothelial cells

$\mathrm{A}, \mathrm{B}$, and C: PPARY and NF-kB by statistical analysis and Western blot analysis and by over-expression of miRNA-130a D: qPCR for miRNA-130a expression

E, F, and G: PPARy and NF-кB by statistical analysis and Western blot analysis and by down-regulation of miRNA-130a.

Negative, negative control group; miRNA-130a, over-expression of miRNA-130a group; anti-130a, down-regulation of miRNA-130a group.

$\# \#<0.01$ comparison with negative group.

HUVECs in vitro model of AS compared with those in the negative group (Fig. 2a-2d). However, down-regulation of miRNA-130a suppressed the levels of IL-18, IL-1 $\beta$, TNF-a, and IL-6 in LPS-induced HUVECs in vitro model of AS compared with those in the negative group (Fig. 2e-2h).

\section{MiRNA-130a regulated PPAR / NF- $\mathrm{kB}$ in HUVECs in vitro}

In this study, we analyzed the mechanism of miRNA-130a in AS. Typically, miRNA-130a mimics was used to up-regulate miRNA-130a expression in LPS-induced HUVECs in vitro model of AS (Fig. 3a). The results of the heat map revealed that over-expres- sion of miRNA-130a reduced the expression of PPARy and induced that of NF-кB in LPS-induced HUVECs in vitro model of AS compared with those in the negative group (Fig. 3b). Moreover, miRNA-130a targeted PPARy, and miRNA-130a over-expression could increase the luciferase reporter activity compared with that in the negative control group (Fig. $3 \mathrm{c}$ and $3 \mathrm{~d}$ ). Over-expression of miRNA-130a suppressed the expression of PPARy compared with that in the negative group (Fig. 3e). Then, miRNA-130a would reduce the PPARy activity protein expression whereas induce that of NF-kB in HUVECs LPS-induced HUVECs vitro model of $A S$, comparison with that in negative group (Fig. 4a-4c). Also, 
the expression of miRNA-130a in HUVECs LPS-induced HUVECs vitro model of AS was reduced by si-miRNA-130a, comparison with that in negative group (Fig. 4d). Our results suggested that down-regulation of miRNA-130a would induce the PPARy activity protein level and suppress that of NF- $\mathrm{kB}$ activity protein level in LPS-induced HUVECs vitro model of AS, comparison with that in negative group (Fig. $4 \mathrm{e}-4 \mathrm{~g}$ ).

\section{Activation of PPAR $y$ diminish the function of miRNA-130a in pro-inflammation effect in LPS-induced HUVECs in vitro model}

The role of PPARy in diminishing the function of miRNA-130a in pro-inflammation effect in LPS-induced HUVECs in vitro model was also explored. Our findings indicated that PPARy $(2 \mu \mathrm{M}$ of GW1929) could promote the protein expression of PPARy and suppress that of NF-kB following miRNA-130a over-expression in LPS-induced HUVECs in vitro model of AS (Fig. S1a-S1c). Besides, activation of PPARy could reduce the effects of miRNA130a on increasing the levels of IL-18, IL-1 $\beta$, TNF- $\alpha$, and IL-6 in the LPS-induced HUVECs in vitro model of AS compared with those in the miRNA-130a group (Fig. S1d-S1g).

Inhibition of NF- $\mathrm{kB}$ diminishes the function of miRNA-130a in pro-inflammation effect in LPS-induced HUVECs in vitro model

The function of NF- $\mathrm{kB}$ in the mechanism of miRNA-130a in pro-inflammation effects in the LPS-induced HUVECs in vitro model of AS was also explored. Our results indicated that si$\mathrm{NF}-\mathrm{k} \mathrm{B}$ could reduce the protein expression of NF- $\mathrm{kB}$ following over-expression of miRNA-130a in LPS-induced HUVECs in vitro model of AS (Fig. S2a, S2b). However, si-NF-кB could reduce the effects of miRNA-130a on increasing the levels of IL-18, IL-1 $\beta$, TNF- $a$, and IL-6 in LPS-induced HUVECs in vitro model of AS compared with that in over-expression of miRNA-130a group (Fig. S2c-S2f). Therefore, these results showed that NF-кB participated in the pro-inflammation effects of miRNA-130a in LPSinduced HUVECs in vitro model of AS.

\section{Discussion}

AS is a systemic disease involving multiple cells, factors, and steps. It is induced by the action of various injury stimulations on the arterial wall, but its pathogenesis remains incompletely illustrated yet (17). miRNA is a class of non-coding small molecular single-strand RNA, which is highly conserved in evolution (about 22 base groups) and can negatively regulate gene expression at the post-transcription level. Multiple studies have verified that miRNA is closely related to AS (15). Typically, plaque formation, development, rupture, and thrombosis are regulated by miRNA (18). Classical AS process can be divided into four stages, including initiation (endothelial activation and inflammation), genesis (sub-intima lipid deposition and foam cell formation), progression (smooth muscle cell proliferation and migration, enlarged necrotic lipid nucleus in the plaque, and angiogenesis), and endpoint (unstable plaque rupture inducing acute coronary artery event) (19). This study demonstrated that miRNA-130a expression was increased in mice with AS. Jia et al. (20) have shown that miR-130a expression in patients with coronary heart disease was down-regulated.

AS is a complicated process involving inflammation, lipid, endocrine, as well as metabolic disorders (21). PPARy is one of the current research hotspots for AS treatment, which can suppress the inflammatory response-related gene transcription (22). In addition, it can antagonize the expression of some inflammatory factors of AS to slow down plaque development. Studies show that although high PPARy expression can be found in the human AS plaque, only a tiny quantity of PPARy expression can be found in the normal artery. Besides, high PPARy expression can be seen in smooth muscle cells during early AS, and its expression quantity persistently increases with disease progression (23). Increased expression can be observed in smooth muscle cells, macrophages, and foam cells, demonstrating that PPARy plays a crucial role during AS process (23). This study showed that over-expression of miRNA-130a reduced PPARy protein expression and stimulated NF-кB protein expression in in vitro HUVECs. Activation of PPARy reduced the pro-inflammation effect of miRNA-130a in the in vitro model of AS. Lin et al. (24) have shown that miR130a suppressed PPARy interaction with 3'UTR of PPARy mRNA in non-small cell lung cancer. This indicates that PPARy suppression by miRNA-130a is important for sensitizing systemic inflammation of AS.

Upregulation of inflammatory factor levels has long been considered to promote AS-related injury. Numerous inflammatory factors, such as TNF-a, are related to AS and other cardiovascular diseases (25). NF-кB is a key factor in the inflammatory response, which can regulate several key enzymes in LDL modification and inflammatory mediator formation in early AS. PPAR is a transcription regulatory factor that participates in lipid metabolism, inflammation, and AS. It includes three subtypes, namely PPARa, $\beta / \delta$, and $y$ (22). Of them, PPARY can suppress inflammatory response and regulate cell proliferation and migration to restrain AS (23). In addition, PPARy ligand or agonist can restrain the activities of monocyte/macrophage transcription factors AP-1 and NF-kB. It can also reduce the inflammatory factor TNF-a to regulate the inflammatory response in AS and prevent AS formation (12). We found that the suppressed NF-кB using si-NF-кB reversed the pro-inflammation effects of miRNA-130a in AS in vitro model. Wang et al. (14) have reported that miR-130a upregulates the mTOR pathway in high-grade serous ovarian carcinoma by regulating NF-кB expression. Our results support a pivotal pro-inflammation function of miRNA-130a in the pathological development of AS by NF-кB/PPAR

\section{Study limitations}

In this study, the sample number of six mice was relatively low and is a limitation. Further studies with larger samples and mores model are warranted. However, the data from our study indicates that miRNA-130a may regulate inflammation of AS. 


\section{Conclusion}

The results of our study demonstrate that miRNA-130a expression was increased in atherosclerotic mice. Over-expression of miRNA-130a increased inflammation factor levels in in vitro HUVECs through the induction of NF-кB expression by PPARy. Therefore, miRNA-130a might be a novel molecular target for AS therapy.

\section{Conflict of interest: None declared.}

Peer-review: Externally peer-reviewed.

Author contributions: Concept - Y.Li; Design - F.L.; Supervision Y.Li; Fundings - Y.Li; Materials - F.L.; Data collection \&/or processing Y.D.; Analysis \&/or interpretation - F.L.; Literature search - Y.Liu; Writing - F.L.; Critical review - Y.Li

\section{References}

1. Mita T, Katakami N, Yoshii H, Onuma T, Kaneto H, Osonoi T, et al.; Collaborators on the Study of Preventive Effects of Alogliptin on Diabetic Atherosclerosis (SPEAD-A) Trial. Erratum. Alogliptin, a Dipeptidyl Peptidase 4 Inhibitor, Prevents the Progression of Carotid Atherosclerosis in Patients With Type 2 Diabetes: The Study of Preventive Effects of Alogliptin on Diabetic Atherosclerosis (SPEADA). Diabetes Care 2016; 39: 139-48.

2. Mamudu HM, Jones A, Paul T, Subedi P, Wang L, Alamian A, et al. Geographic and Individual Correlates of Subclinical Atherosclerosis in an Asymptomatic Rural Appalachian Population. Am J Med Sci 2018; 355: 140-8.

3. Randrianarisoa $E$, Lehn-Stefan A, Wang $X$, Hoene $M$, Peter A, Heinzmann SS, et al. Relationship of Serum Trimethylamine N-Oxide (TMAO) Levels with early Atherosclerosis in Humans. Sci Rep 2016; 6: 26745.

4. Santulli G. microRNAs Distinctively Regulate Vascular Smooth Muscle and Endothelial Cells: Functional Implications in Angiogenesis, Atherosclerosis, and In-Stent Restenosis. Adv Exp Med Biol 2015; 887: 53-77.

5. Menghini R, Casagrande V, Federici M. MicroRNAs in endothelial senescence and atherosclerosis. J Cardiovasc Transl Res 2013; 6: 924-30.

6. Novák J, Olejníčková V, Tkáčová N, Santulli G. Mechanistic Role of MicroRNAs in Coupling Lipid Metabolism and Atherosclerosis. Adv Exp Med Biol 2015; 887: 79-100.

7. Li ZC, Han N, Li X, Li G, Liu YZ, Sun GX, et al. Decreased expression of microRNA-130a correlates with TNF- $\alpha$ in the development of osteoarthritis. Int J Clin Exp Pathol 2015; 8: 2555-64.

8. Yao L, Guo Y, Wang L, Li G, Qian X, Zhang J, et al. Knockdown of miR-130a-3p alleviates spinal cord injury induced neuropathic pain by activating IGF-1/IGF-1R pathway. J Neuroimmunol 2021; 351: 577458.

9. Ortuño Sahagún D, Márquez-Aguirre AL, Quintero-Fabián S, LópezRoa RI, Rojas-Mayorquín AE. Modulation of PPAR-y by Nutraceutics as Complementary Treatment for Obesity-Related Disorders and Inflammatory Diseases. PPAR Res 2012; 2012: 318613.
10. Liao Z, Dong J, Wu W, Yang T, Wang T, Guo L, et al. Resolvin D1 attenuates inflammation in lipopolysaccharide-induced acute lung injury through a process involving the PPARy/NF-кB pathway. Respir Res 2012; 13: 110.

11. Liu L, Liu J, Niu G, Xu Q, Chen Q. Mycobacterium tuberculosis 19$\mathrm{kDa}$ lipoprotein induces Toll-like receptor 2-dependent peroxisome proliferator-activated receptor y expression and promotes inflammatory responses in human macrophages. Mol Med Rep 2015; 11: 2921-6.

12. Chen $\mathrm{Y}$, Zhao $\mathrm{H}$, Ren $\mathrm{X}$. Estrogen and progestogen inhibit NF-кB in atherosclerotic tissues of ovariectomized ApoE (-/-) mice. Climacteric 2016; 19: 357-63.

13. Zhou F, Liu D, Ning HF, Yu XC, Guan XR. The roles of p62/S0STM1 on regulation of matrix metalloproteinase- 9 gene expression in response to oxLDL in atherosclerosis. Biochem Biophys Res Commun 2016; 472: 451-8.

14. Wang Y, Zhang X, Tang W, Lin Z, Xu L, Dong R, et al. miR-130a upregulates $\mathrm{mTOR}$ pathway by targeting TSC1 and is transactivated by NF-кB in high-grade serous ovarian carcinoma. Cell Death Differ 2017; 24: 2089-100

15. Wang X, Huang R, Zhang L, Li S, Luo J, Gu Y, et al. A severe atherosclerosis mouse model on the resistant NOD background. Dis Model Mech 2018; 11: dmm033852.

16. Livak KJ, Schmittgen TD. Analysis of relative gene expression data using real-time quantitative PCR and the 2(-Delta Delta C(T)) Method. Methods 2001; 25: 402-8.

17. Zhang JY, Gong YL, Li CJ, Oi Q, Zhang QM, Yu DM. Circulating MiRNA biomarkers serve as a fingerprint for diabetic atherosclerosis. Am J Transl Res 2016; 8: 2650-8.

18. Yilmaz SG, Isbir S, Kunt AT, Isbir T. Circulating microRNAs as Novel Biomarkers for Atherosclerosis. In Vivo 2018; 32: 561-5.

19. Mao Z, Wu F, Shan Y. Identification of key genes and miRNAs associated with carotid atherosclerosis based on mRNA-seq data. Medicine (Baltimore) 2018; 97: e9832.

20. Jia QW, Chen ZH, Ding XQ, Liu JY, Ge PC, An FH, et al. Predictive Effects of Circulating miR-221, miR-130a and miR-155 for Coronary Heart Disease: A Multi-Ethnic Study in China. Cell Physiol Biochem 2017; 42: 808-23.

21. Xiong W, Zhao X, Villacorta L, Rom O, Garcia-Barrio MT, Guo Y, et al. Brown Adipocyte-Specific PPARy (Peroxisome Proliferator-Activated Receptor y) Deletion Impairs Perivascular Adipose Tissue Development and Enhances Atherosclerosis in Mice. Arterioscler Thromb Vasc Biol 2018; 38: 1738-47.

22. Shi ST, Li YF, Guo YQ, Wang ZH. Effect of beta-3 adrenoceptor stimulation on the levels of ApoA-I, PPARa, and PPARy in apolipoprotein E-deficient mice. J Cardiovasc Pharmacol 2014; 64: 407-11.

23. Gao LN, Zhou X, Lu YR, Li K, Gao S, Yu CQ, et al. Dan-Lou Prescription Inhibits Foam Cell Formation Induced by ox-LDL via the TLR4/ NF-кB and PPARy Signaling Pathways. Front Physiol 2018; 9: 590.

24. Lin L, Lin H, Wang L, Wang B, Hao X, Shi Y. miR-130a regulates macrophage polarization and is associated with non-small cell lung cancer. Oncol Rep 2015; 34: 3088-96.

25. Liu M, Yu P, Jiang H, Yang X, Zhao J, Zou Y, et al. The Essential Role of Pin1 via NF-кB Signaling in Vascular Inflammation and Atherosclerosis in ApoE-/- Mice. Int J Mol Sci 2017; 18: 644. 


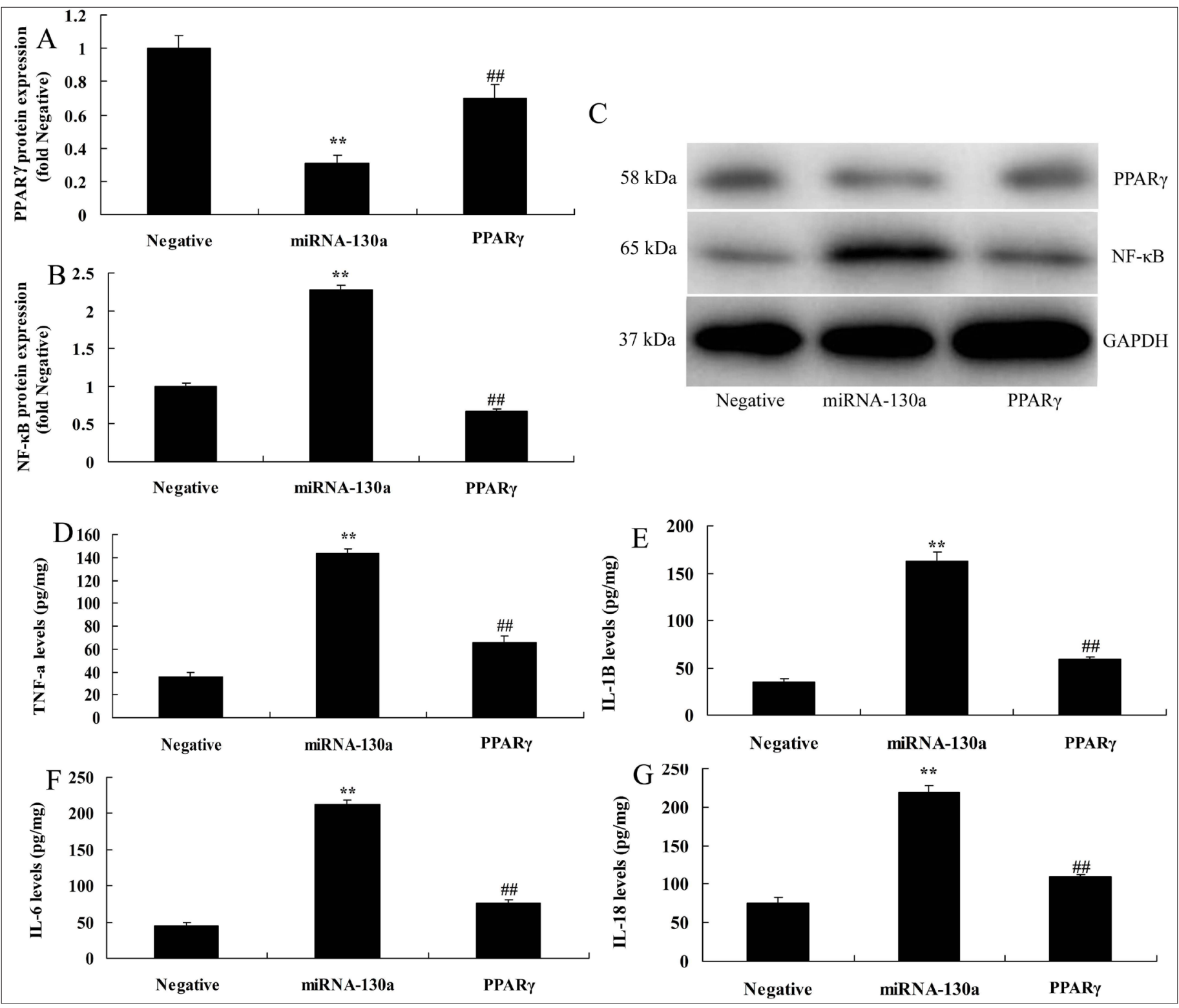

Figure S1. Activation of PPARy reduced the effect of miRNA-130a on inflammation in in vitro model $A, B$, and C: PPARy and NF-kB by statistical analysis and Western blot analysis $\mathrm{D}, \mathrm{E}, \mathrm{F}$, and G: TNF- $a, \mathrm{IL}-1 \beta$, IL-6, IL-18 levels

Negative, negative control group; miRNA-130a, over-expression of miRNA-130a group; PPARy, over-expression of miRNA-130a and PPARy group. $\# \# P<0.01$ comparison with negative group, ${ }^{* *} P<0.01$ comparison with over-expression of miRNA-130a group. 


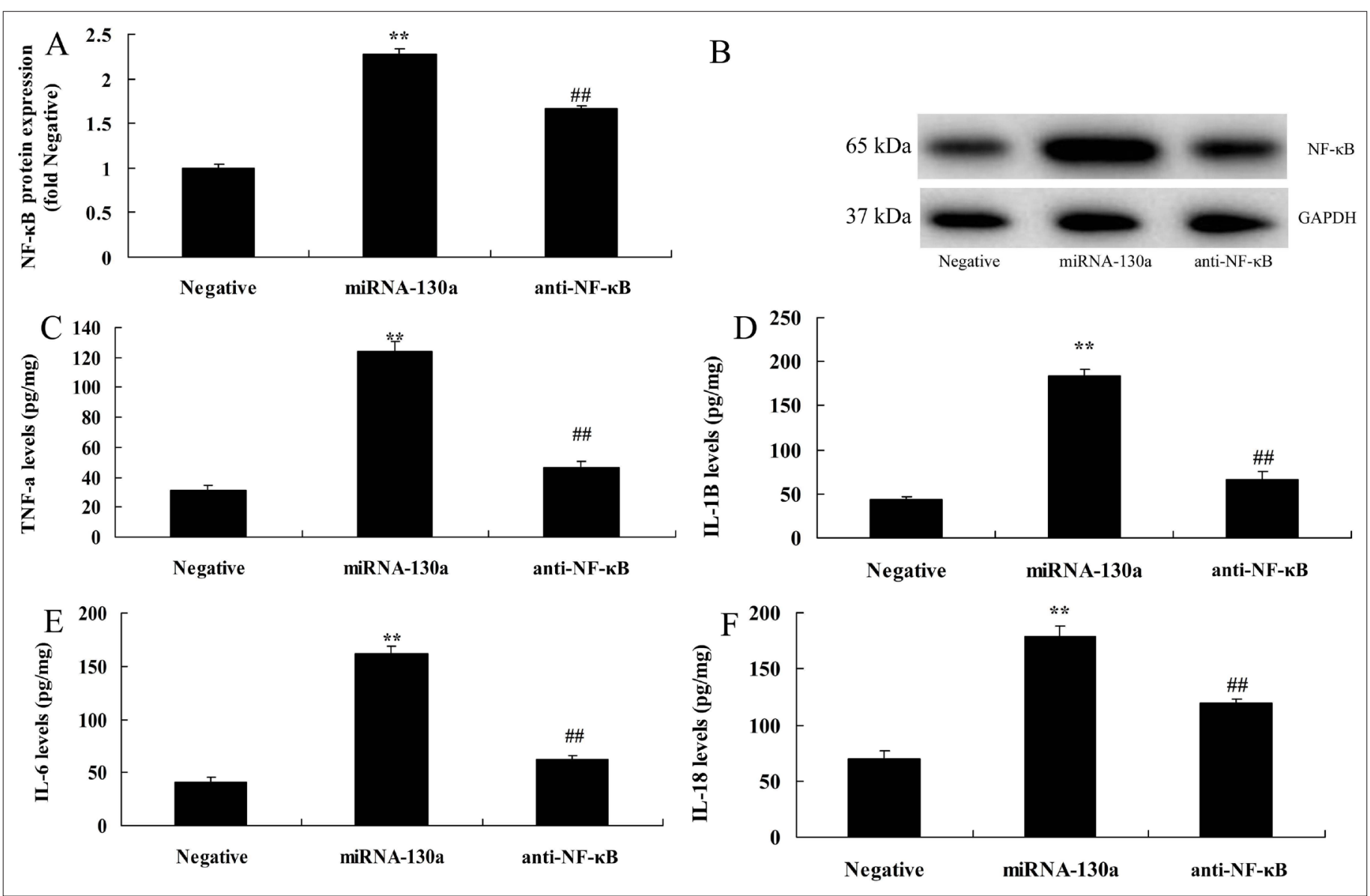

Figure S2. Inhibition of NF- $\mathrm{kB}$ reduced the effect of miRNA-130a on inflammation in in vitro model $A$ and $B: N F-\kappa B$ by statistical analysis and Western blot analysis

C, D, E, and F: TNF- $\alpha$, IL-1 $\beta$, IL-6, IL-18 levels

Negative, negative control group; miRNA-130a, over-expression of miRNA-130a group; anti- NF-кB, over-expression of miRNA-130a and si-NF-кB group. $\# \# P<0.01$ comparison with negative group, ${ }^{* *} P<0.01$ comparison with over-expression of miRNA-130a group. 